\title{
Data Wrangling- A Goliath of Data Industry
}

\author{
Ritvik Voleti \\ KCC-ITM Greater Noida
}

\begin{abstract}
Analyzing data in any industry along with the prospect of smartly utilizing data through special technology brings with it an ocean of opportunities. Nonetheless it is cumbersome task to modify and collect data as expected by the user. Data cleaning's importance cannot be overstated enough but it takes a lot of precious time and important resources. Data Wrangling is much more than just modifying and cleaning data and provides user the benefit of interactive and an efficient data. It is a method in which we have data identification, extracting, cleaning and integrating data for a dataset which would be analyzed as needed. Even though tools are in abundance, but software solutions are being a rarity. We have keenly discussed about the various aspects of data wrangling, munging data. There is a wide variety of ETL tools and mediums, but it needs manual effort in presence of technical experts for every step in the process. We start by the topic overview with the present issues, tangible mutual commands along with a discussion on software resolutions, various methods.
\end{abstract}

Keywords : Data wrangling, ETL, SQL

\section{INTRODUCTION}

Data is referred to as raw facts and figures without any meaning. Data with no meaning is of no use hence we have to analyze it to enhance the meaningfulness of the data to make it into information. Meaningful data is called information. Bernardmar said that even though the small businesses have data limitedness it doesn't bring the IT MNC's for instance Google, Twitter into an undue advantage in the big data industry and continued saying that small companies have to be up their game to compete with them with upmost observation[1].

An old Naval Officer made from a huge quantity of traditional writings of collection to humungous joined coordinated constructed routes. This was the main conversion of human understandable material to machine understandable mechanism. By processing the routes he decreased the aggregate Navy travel by almost thirty-three percent. The majority of people agree upon the fact that data analysis is an integral part in making key resolutions. A small but an important percentage (16\%) of people feel that the biggest advantage is the improvement in decisive initiatives[2]. For enhancing the lifestyle as a whole, data plays a key role in that. It amplifies qualities which emphasizes the need to utilize more and more data. Data provides reasonability for both right and wrong accord to the shareholders. Irrespective of the policies and the result you expected. Data provides examining the condition of essential organizational systems: With the efficient usage of data for examining the quality, difficulties are faced head on by organizations preventing a big crisis like the one we are facing these days. Data is equal to Knowledge. It encourages problem tracking in the companies to identify the root cause of the problem. It also provides companies to simulate correlation with whatever is occurring at various places, divisions and structures. Companies can know about in-depth analysis of minute details of the things happening within the company.

\section{DEFINING DATA WRANGLING WITH EXPLANATIONS}

The method of using unorganized, meaningless (in itself), complicated data and cleaning it along with shortening the data to make it accessible to approach, arrange and scrutinize accordingly is referred to as data wrangling. For any latest dataset one must analyze main factors like magnitude, encoding along with the configuration[3]. Applying practicality in the concept, which would mean analyzing a particular area, rows and columns inside a dataset and performing a function like combining, parsing, cleaning, compensating, and removing waste data to build a much needed result that would prove useful later on. The data which is wrangled has the potential in industries, architecture of data, data scientists whose responsibility is based on processing the data continuously and replenishing it in various forms. We have seen a major shift in the last few years with more emphasis on data wrangling as it is transforming into a rapidly flourishing part of the data industry. Unordered and complicated datasets were considered a difficulty in the analyzation of data, but on the contrary the capability to modify data has revamped the actions, making it from a tedious, time-taking attempts to master different origins of data.

\section{SIGNIFICANCE OF DATA WRANGLING}

Computers depend on traditional data (key part in ML) which encourages it to train and improve its AI. Hence, the algo's won't extract any sort of needed pattern when our data is messy, not meaningful and contains questionable content. "Garbage In Garbage Out" approach is utilized in ML. Data cleaning and appropriate preparation is essential, otherwise we are prone to a situation in which our data models would provide unpredictably faulty decisions which has the potential to influence the consumers and their revenue. Therefore, understanding the flaws of our data is key whenever we utilize it like an input and it also helps in predicting about our analysis. Even though data processing in data wrangling is unmotivating, it is essential as it helps in right utilization of data. Data wrangling has the potential to supply data value by analysis, or taken inside a collaboration along with workflow gadgets for driving downstream steps as and when it is followed up at destination form. 
Conformance (changing) contrasting data components to the exact form directs to the disadvantage in siloed data. It is highly important in the case of multi data sources that are expected to wrangle in the same style. For instance with the help of data wrangling we are able to analyze different athletes speeds while sprinting based on conforming the format regardless of the time and stadium. Data wrangling ensures data organization in a standard manner and iterative process which converts different data sources in a same format which adds to it being more reusable for many times. As and when data conformation is completed in a standardized style, it makes one in a strong condition for understanding cross-dataset analysis.

\section{OBSTACLES SURROUNDING DATA WRANGLING}

About eighty percent of time is wasted in obtaining value by big data by data wrangling contrarily to the data analytics[4]. Therefore effectiveness in data wrangling has to improve. Till date, the difficulties of big data with data wrangling are met in the extent of individual parts like extracting data/integration. Still distributing knowledge in the parts which have the highest capability to enhance data wrangling procedure[5]. These challenges are satisfied only at individual level[6].

- Direct access of required data is efficient for any data scientist, data analyst. Or else, we have to submit concise commands to get "scrubbed" data, with the aim for granting the request and proper execution. Navigation of policies barrier is tough and time taking.

- Machine Learning experiences leakage in data that is an enormous challenge to deal with. The dangers increases gradually as Machine Learning algos are utilized in data handling. Accuracy in data is an integral part of prediction. When the estimated calculation is done from an ambiguous data the calculation is like a random guess. As a result of imprecise algorithm with wrong calculations will disturb the functioning of various companies.

- Recognizing the need of scaling the queries [7] which are accessible with proper indexing bring in a challenge[8]. Completely analyzing the correlation is key before building a model. Repeated unnecessary data has to be removed prior to the examining the relation to the final result. Avoiding this would prove fatal later on. Regularly, in large data of files, a group of columns that are closely linked, hence showing that containing redundant data, that only provides in featuring and making selecting the model tougher. Even though these repeated-ness will provide a large correlation coefficient even though occasionally it would not.

- Some key issues need to be brought up. For instance, various quality evaluations are not in limit [9] and examining even basic queries utilized in mappings would need large updates to traditional expectations in case of large dataset. A dataset lacks usually values, has mistakes, noise. Soapy Eye, Inadvertent Mislabeling, Technical Faults are some of its causes. Its affect on the class of data processing task is familiar and causes in inferior results and later on causes poorly managed business action. Messy, unrealistic data in ML algorithms is like adding salt to wounds. A trained dataset algo it could prove unusable for its needs.

- Reproducibility, Documentation are essential but is usually an ignored part of any research. Data handling and procedures with time along with the regeneration of past acquired conclusions are mutual needs that is difficult to sustain, specially in mutually interactive connectivity.

- Selection Bias is not given due importance till we see a failed model. It cannot be neglected especially in data science. It is essential to ensure that the given training data model is demonstrative of the operating model. Ensuring with proper weights in bootstrapped designing needs structuring a design for only this usage.

- Data combining along with combining data integration is usually essential to build the image. Hence, combining, linking the diverging designs, coding protocols, rules, modelling data is an essential need as we prepare data for using later on. Changing cases can be recognized on the basis of different origins, data types, linking methodology, reasonability for data combining.

- The relevance of any entity is paramount. For instance the key named "customer". What is the identity of this customer? There is a possibility of having a User $I D$ within a table but if the same user goes and comes back, this could make a duplicate User ID for them. Are Cristiano Ronaldo, C.Ronaldo, and C.R considered the same user? Are the users same if they share an account with multiple users? Is masking identities identifiable? Is a same account utilized by the different family members of the user? The ambiguities are everlasting and need to be addressed.

\section{WAYS FOR EFFECTIVE DATA WRANGLING}

With this sort of analysis we grasped a keen insight on data quality along with querying, reasoning the data. The components discussed above are integral for a efficient data wrangling. Systems for extracting data with ease and efficiency are available[10]. Two concepts analyzed in depth is data integration [11], exchange of data[12]. There is a sense of vulnerability in case of limited framework within data extraction[13] and examination is needed to ensure that tools used in extraction are approachable to high quality analysis with ideas coming from data integration, feedback from the user. Present ideas and transitional data cleaning procedure, data integration is 
useful potentially in enhancing wrapped inductive value[14].

- Manually wrangling data (data munging) provides us for opening, inspecting, cleansing, manipulating, testing and data distribution manually. Initially it would provide plenty of fast and unrelatable data. But, this technique is not encourage able as it lacks efficiency. This technique is vital in case of single with current analysis cases. Continuing this process for long term takes a lot of time, susceptible to error due to human involvement. This technique always brings with it the chance of ignoring an important phase and resulting with improper data for the consumers.

- To make matters better we have programme based gadgets which has the potential of improving data wrangling process. SQL is a great example for semiautomated technique. One must extract in data from the source inside a table, this brings with it an improved position for data profiling and puts one in an improved condition for data profiling, and analyzing the inclinations, editing it, and executing data and providing summary from queries inside it as compared to a spreadsheet. Also, having a repetitive command which has a limited data origins, one has the potential to build a process in SQL for analyzing one's data wrangling.

A further advancement compared to the stored processes is the ETL tools. ETL's take out data by the source form, modify it for matching the resultant format, thereby loading the data in resultant area. Extraction-Transformation-Load has a wide range of tools up its sleeve. Few of them are free of cost. These tools provides us with an update compared to Standard Query Language stored queries as the data handling is more efficient and simply better. ETL's are more efficient in composite transformations, lookups. Also they have a better memory management capability that is highly needed especially in big datasets.

- When the requirement is there for redundant and compound data wrangling there has to be a serious consideration of building company warehouse of data with the help of fully automated workflows. The following technique brings with it, data wrangling with a reusable and automated mentality. This process then runs in a automating plan for current data load by a present data origin in a adapted format. Even though this technique needs better analysis, framework and modifications, along with current data maintenance, governance, it supplies the advantages of re-utilizing ExtractionTransformation-Load logic, and we can rework the adapted data in variety of company cases.

Data wrangling is vital in any company analysis and just cannot be neglected. Ideal scenario for managing ones disruptive data is building schedule automated based tasks to get the maximum from data wrangling, adapt dissimilar data parts in a similar format saving the analysts time to provide a improved data combined commands.

Ways to enhance data wrangling pace:

These methods are encouraging but we need to focus on speeding up the vital data wrangling process. Speed in data wrangling is something which we cannot afford to lose hence appropriate steps need to be made to enhance performance.

- At any interval it is tough to highlight on the required needs to the important issues to be addressed. Quick results also would be needed. The ideal method to withstand these issues is discussed later. We must find the ideal solution to each issue by isolating the problem. We must build some parameters of high importance and address them with more importance. We must keep track of tasks and solutions as it would fasten the process of building a proper approach.

- Assimilating data professionals other than IT sector epitomizes a move which todays businesses are not encouraging which has caused is a move that modern day businesses have stopped doing and that has lead to the challenges faced. Even though data thrives for analyzation but it is depended on the function of a specialist by modelling our data, data value apart from data about data.

- There has to be a encouragement to be in a connected society and analyze various case studies of your particular industry. The ideal case to improve is analyzing your colleagues performances. Joining communities which have mutual welfare could quicken the learning. We gain a lot of familiarity alongside community that are motivated to prosper their own careers within data science with an attitude of constantly learning and improving daily. With time we know more learning from different cases analyzing them. They can be very vital.

- Each crew in any company has an individual aim and objectives. However, mostly they are share one common goal. Collaboration along with other teams be it engineering, data science, different departments within a team could also prove underrated but highly important. It brings with it a different mindset. Many times we are static and a hint of shift in perspective is all that we need. For instance, the requirement to comprehend user problems could belong in the gadget development team, not so in the minds of operations crew as it could shorten up time utilized in the logistics. Collaboration hence could quicken the speed of identifying the ideal dataset. This provides with it the perfect decisions with it. An inclusive dataset is offered which is quite innovative. 
- An unforgettable cause in delay, errors in data is produced due to data mapping and which is highly difficulty in case of data wrangling. A solution for this issue is messing about with data. It does not look like a viable option but on the contrary this reduces investing wasting hours to map our data. Data laboratories are vital in scenarios in which an analyst has the chance to utilize possible data feeds along with variables for learning about if they are projecting or essential in analyzing or model the data.

- Data wrangling when utilized to gain user visions with the assistance of Facebook, Twitter or any other social media, surveys, comment section improves the knowledge of how to use the data properly like user retaining. But, the difficulty ascends when finally the usage for data wrangling isn't identified. The definite result extracted using data wrangling would be a disappointing one. Hence, it is imperative to extract the final aim via data wrangling and not forgetting to quicken the process.

- Intelligent awareness has the extracting capability to provide responses for data wrangling problems. We have to understand whether scalability along with granularity is maintained and respond accordingly. Try to figure out the method to utilize similar datasets in varying time spans. Find the appropriate gadgets or tools to decrease the time utilized in data wrangling. We must understand that are we able to put in the proper structure needed with least changes. We have to analyze insights to improve data wrangling.

- The vital aspect of any industry is to locate main data for making crucial decisions at right moments. There is no room for randomness or complacency, and absolute conciseness in data is essential for any successful business.

Big Data in general is quite difficult to completely understand, considering the amount of data. Even though the amount of data that is characterized largely dependent due to the usage of handling and evaluating large amount of datasets, it requires more important technologies[15].

\section{CONCLUSION}

Finally, this shows the boldness in data wrangling process. We analyze many solutions for different issues and sometimes even special cases are in existence but none of the solutions cover up all the issues. Further analysis or research is needed for combating the difficulties in data wrangling with proper tools. This process presents with its own set of challenges and improvements. Fully recognizing the quality of data, reproducible process, data management cannot be neglected. The focus has to shift in building a data wrangling process which saves money, as this would balance the different unrealistic tasks to make them in a closer reach. This paper tries to analyze data wrangling which has room for improvement in working where till date further investments and support is needed. This review has pin-pointed some key difficulties in research which are brought up using data wrangling. There is immense potential for further analysis and discussion in this diverse and exciting process.

\section{REFERENCE}

[1] https://www.bernardmarr.com/default.asp?contentID=1442

[2] Deloitte, The Analytics Advantage We're just getting started, https://www2.deloitte.com/content/dam/Deloitte/global/Documen ts/Deloitte-Analytics/dttl-analytics-analytics-advantage-report061913.pdf

[3] Florian Endel, Harald Piringer, "Data Wrangling: Making data useful again" University of Technology Vienna VRVis Research Center, Vienna, Austria.

[4] Lohr, S.: For big-data scientists, 'janitor work' is key hurdle to insights. The New York Times (2015), http://nyti.ms/1 Aqif2X.

[5] Furche, T., Gottlob, G., Libkin, L., Orsi, G., Paton, N.W.: Data wrangling for big data: Challenges and opportunities. In: EDBT (2016)

[6] Tim Furche, Georg Gottlob, Bernd Neumayr, and Emanuel Sallinger, Data Wrangling for Big Data: Towards a Lingua Franca for Data Wrangling, University of Oxford.

[7] M. Armbrust, E. Liang, T. Kraska, A. Fox, M. J. Franklin, and D. A. Patterson. Generalized scale independence through incremental precomputation. In SIGMOD, pages 625-636, 2013.

[8] W. Fan, F. Geerts, and L. Libkin. On scale independence for querying big data. In PODS, pages 51-62, 2014.

[9] P. Bohannon, W. Fan, M. Flaster, and R. Rastogi. A cost-based model and effective heuristic for repairing constraints by value modification. In SIGMOD, pages 143-154, 2005.

[10] Furche, T., Gottlob, G., Grasso, G., Guo, X., Orsi, G., Schallhart, C., Wang, C.: DIADEM: thousands of websites to a single database. PVLDB 7(14) (2014)

[11] Lenzerini, M.: Data integration: A theoretical perspective. In: Popa, L., Abiteboul, S., Kolaitis, P.G. (eds.) PODS. pp. 233-246. ACM (2002)

[12] Fagin, R., Kolaitis, P.G., Miller, R.J., Popa, L.: Data exchange: semantics and query answering. Theor. Comput. Sci. 336(1), 89124 (2005)

[13] S. Chuang, K. C. Chang, and C. X. Zhai. Collaborative wrapping: A turbo framework for web data extraction. In ICDE, 2007.

[14] S. Ortona, G. Orsi, M. Buoncristiano, and T. Furche. Wadar: Joint wrapper and data repair. PVLDB, 8(12):1996-2007, 2015.

[15] Hu, H., Wen, Y., Chua, T.S., and Li, X. (2014). Toward scalable systems for big data analytics: A technology tutorial. IEEE Access, 2, 652 \{687. doi: 10.1109/ACCESS.2014.2332453. 